\title{
DISEÑO Y FABRICACIÓN DIGITAL DE TARJETAS POP-UPS EN ENTORNOS EDUCATIVOS MEDIANTE TECNOLOGÍAS DE BAJO COSTE
}

\author{
DESIGN AND DIGITAL MANUFACTURE OF POP-UP CARDS IN EDUCATIONAL \\ ENVIRONMENTS USING LOW-COST TECHNOLOGIES.
}

\begin{abstract}
Alejandro Bonnet de León; alebonle@hotmail.com; (iD orcid.org/0000-0001-7992-545X
Jose Luis Saorin; jlsaorin@ull.edu.es; iD orcid.org/0000-0003-3240-3317

Jorge de la Torre-Cantero; jcantero@ull.edu.es; iD orcid.org/0000-0001-5516-0456

Cecile Meier; cemeier@ull.edu.es; (iD orcid.org/0000-0001-7160-2154

Eliseo García Marrero; eliseogarmar@gmail.com; (iD orcid.org/0000-0002-7576-019X
\end{abstract}

Universidad de la Laguna (España)

\section{Resumen}

La implantación de tecnologías de fabricación digital en centros escolares requiere habitualmente la creación de espacios específicos (Makerspaces, Fab labs, etc.). Sin embargo resulta interesante la introducción de estas tecnologías en aulas tradicionales. En este artículo se describe una experiencia llevada a cabo en el Colegio San Isidro, Los Salesianos de la Orotava, Tenerife en 4으 curso de secundaria en la asignatura de expresión plástica, visual y audiovisual, en el que se ha realizado una actividad de diseño y creación de tarjetas pop-up. La realización de esta actividad implica el corte y doblado de papel que habitualmente se realiza mediante tijeras o cutter. Sin embargo, con la aparición de técnicas de fabricación digital de bajo coste, este proceso puede ser automatizado e introducido en cualquier aula.

Palabras clave: Fabricación digital, educación, pop-up, plotter de corte, vectorización.

\section{Abstract}

The implementation of digital manufacturing technologies in schools usually requires the creation of specific spaces (Makerspaces, Fab labs, etc.). However, it is interesting to introduce these technologies in traditional classrooms. This article describes an experience carried out at Colegio San Isidro, Los Salesianos de la Orotava, Tenerife in the 4th year of secondary school in the subject of plastic, visual and audiovisual expression, in which an activity of design and creation of pop-up cards has been carried out. This activity involves cutting and folding paper, which is usually done with scissors or a cutter. However, with the emergence of low-cost digital manufacturing techniques, this process can be automated and introduced into any classroom.

Keywords: Digital manufacturing, education, pop-up, cutting plotter, vectorize. 


\section{INTRODUCCIÓN}

Las tecnologías de fabricación digital incluyen el proceso de creación, edición y manejo de ficheros digitales (Alvarado, 2012). Hasta hace unos años todos estos procesos necesitaban una formación especializada, habitualmente en el ámbito de la ingeniería y la arquitectura. Sin embargo, con la evolución del software y del hardware, estas tecnologías se han hecho accesibles, tanto en lo económico como en los aspectos formativos, con lo que se pueden realizar los procesos ligados a la fabricación digital de forma sencilla (Bonnet de León, Meier, Saorín, de la Torre-Cantero, \& Carbonell, 2017). Por lo tanto, la incorporación de estas tecnologías en entornos educativos es una opción viable.

Dentro de los distintos informes internacionales sobre educación existe uno específico sobre tecnologías que se ha convertido en un referente: el "Informe Horizon". En este informe, concretamente el que analiza las nuevas tecnologías en secundaria y Bachillerato, la creación de laboratorios de fabricación digital (Ilamados también Fablabs o Makerspaces) se incluye desde el año 2015 y se prevé que sea una realidad menos de un año (Freeman, Becker, \& Cummins, 2017). Como también señala dicho informe, su implantación en centros de enseñanza primaria y secundaria, es ya una realidad en casos como el Frysklab, el taller creativo de la biblioteca municipal de Fryslan (Holanda), el Makerspace del Centro de Enseñanza Secundaria de Sierra Vista de La Puente, en California, o en el Centro de Enseñanza Secundaria de Monticello, en Charlottesville en Virginia, aportando espacios de trabajo para la investigación tecnológica con beneficios tangibles como el descenso del ausentismo escolar, la mejora en materias como matemáticas o su interés por carreras de ciencias o ingenierías, a partir del uso de herramientas de fabricación digital. En España, Galicia implantará el próximo año el primer Bachillerato STEMbach (de excelencia en ciencias y tecnologías) que incluyen espacios Maker (Europa Press, 2018).

El aprendizaje del diseño y la fabricación digital también está empezado a ser reconocida en organismos oficiales. Por ejemplo, a nivel local, la Consejería de Economía, Industria, Comercio y Conocimiento del Gobierno de Canarias, en julio de 2016, en su convocatoria de subvenciones orientadas a la mejora de las competencias digitales de la población residente en Canarias, incluye diseño, modelado y fabricación digital entre las tres temáticas de la convocatoria (BOC, 2016). Como consecuencia de esta iniciativa la Consejería de Educación en Canaria ha creado el programa "STEAM de fomento de vocaciones científicas y creatividad". Dicho programa incluye actividades formativas, ligadas al diseño y la fabricación digital, dirigida a educación secundaria y bachillerato. En concreto en el año 2018 se han creado dos Fab Labs, uno en el Centro de Profesorado de La Laguna y otro en el de Las Palmas de Gran Canaria y se pretenden crear otros ocho, repartidos en las siete islas. Además, este programa está apoyando la creación de Aulas Maker en los centros de secundaria. Para llevar a cabo proyectos educativos, en estos laboratorios se dispone de maquinaria de fabricación, como impresoras 3D y máquinas de corte láser (Gobierno de Canarias, 2018).

Sin embargo a pesar de estos avances, todavía resulta difícil disponer de un laboratorio de fabricación digital o aula maker en el propio centro escolar. Debido a esto, es interesante 
plantear actividades que introduzcan la fabricación digital en cualquier aula. En el entorno educativo, hay centros que han implementado impresoras 3D en el aula y realizan investigaciones acerca del alcance que esta herramienta de fabricación digital tiene en los alumnos (Saorín, de la Torre-Cantero, Bonnet de León, \& Meier, 2016; de la Torre-Cantero, Saorín, Meier, Melián Díaz, \& Alemán, 2015). A pesar de que los resultados son aceptables, los tiempos de trabajos de las impresoras 3D suelen sobrepasar el tiempo disponible en sesiones ordinarias dentro del horario lectivo.

En este sentido, la fabricación mediante máquinas de corte, puede ser una alternativa para evitar los problemas del excesivo tiempo que requieren las impresoras 3D. Este proceso de fabricación si bien no es exactamente igual que la impresión 3D, puede ser una opción más factible en tanto a los tiempos necesarios para realizar piezas de gran tamaño o un mayor número de ellas.

La construcción de piezas mediante máquinas de corte (fresadora, máquina de corte láser y plotter de corte) es mucho más rápida pero no existen muchos antecedentes de su uso en entornos educativos como por ejemplo en secundaria y bachillerato. Esto es debido principalmente a su alto coste, ruido o peligrosidad. Sin embargo, los avances tecnológicos están permitiendo resolver estos inconvenientes y por lo tanto, se pueden empezar a diseñar actividades de fabricación por corte automatizado en aulas tradicionales con estas herramientas. En este artículo se plantea una actividad de diseño y fabricación digital de bajo coste que utiliza programas gratuitos y un plotter de corte portátil de bajo coste, que permiten transferir las experiencias de los laboratorios de fabricación digital a cualquier centro educativo.

La actividad propuesta consiste en la elaboración de tarjetas pop-up por parte del alumnado, mediante un plotter de corte y programas de dibujo vectorial online. El objetivo de la investigación es comprobar si para impartir los contenidos de forma y color, se pueden implementar el uso de programas vectoriales y herramientas de fabricación digital en el aula. Para validar este objetivo, Se ha desarrollado una prueba piloto con 63 alumnos de 4ㅇ de la ESO del Colegio San Isidro, Salesianos en La Orotava, en el marco de la asignatura de expresión plástica, visual y audiovisual, en la que se han incorporado estas tecnologías. Se ha recurrido a una metodología cuasi-experimental donde se ha utilizado como grupo experimental los alumnos que cursaban el cuarto curso de secundaria en la asignatura de expresión plástica, visual y audiovisual en el momento de la experiencia. Los resultados en la experiencia demuestran que la fabricación digital mediante plotter de corte y el diseño vectorial mediante programas digitales online resultan viables en educación secundaria y se valoran de forma positiva por parte de los alumnos. 


\section{ANTECEDENTES}

\subsection{Actividades Papel recortado (kirigami) en entornos escolares}

La creación de tarjetas pop-up entra en el campo del kirigami. El término kirigami deriva de las palabras japonesas KIRU (cortar) y KAMI (papel). Hay muchas formas de kirigami, pero el más extendido es aquel que se dobla un papel por la mitad, se corta una forma y se genera una pieza tridimensional al abrirse (Temko, 1962). Dicha técnica también se conoce como pop-up (Carter \& Díaz, 1999; Washington Patente no 5,450,680, 1995).

Al igual que el papel, se estima que el Kirigami nació en China y fueron usados en la vida cotidiana como decoraciones festivas o como motivos religiosos, pero también se usaban algunos de estos diseños como patrones para realizar decoraciones en tejidos y cerámicas expresando principios morales, filosofías e ideales estéticos (Jablonski, 1976). El ejemplo más antiguo de esta actividad que sobrevive, es un círculo simétrico, encontrado en Xinjiang, China, datado en el siglo XI (McCormick \& White, 2011). Este también fue inicialmente un conocimiento que se transmitió de forma oral hasta que en 1721, aparece la primera referencia publicada sobre plegado y corte de papel, en un libro japonés de Kan Chu Sen (Sen, 1721).

El kirigami se ha usado tradicionalmente como técnica de decoración o entretenimiento lúdico. Sin embargo también se utiliza en diseño arquitectónico (Vyzoviti, 2006), se han encontrado aplicaciones del kirigami en la creación de estructuras sólidas a microescala y metamateriales con grapheno (Blees, y otros, 2015). En otras investigaciones se fabrican nanocompuestos de kirigami como electrodos de plasma que abren una amplia gama de soluciones tecnológicas para dispositivos electrónicos (Shyu, y otros, 2015). También se testea la elasticidad de los materiales como por ejemplo en baterías, aplicando técnicas de kirigami (Isobe \& Okumura, 2016). Incluso se crean músculos artificiales mediante el diseño de Kirigami (Sareh \& Rossiter, 2012).

En el contexto educativo se usa el corte y doblado de papel de manera habitual. Kobayashi y Yamada (2013) presentan el uso del origami y kirigami en un curso que tuvo como objetivo emplear estas técnicas como recurso lúdico y educativo, al mismo tiempo que se buscó introducir aspectos culturales del arte de doblado y corte de papel. En otras investigaciones se propone aumentar las habilidades de visualización espacial a través del origami, el kirigami y la arquitectura origámica (Nogueira Valente \& Yukari Ota, 2015). También se utiliza para el aprendizaje de los fractales (fig. 1) en las matemáticas (García Cuéllar, Flores Salazar, \& Martínez Miraval, 2017), para mejorar las habilidades creativas (Castillo Cordova, 2016), para incentivar el pensamiento creativo (Romero Velasco \& Tovar Cifuentes, 2018), para enseñar sistemas micro electromecánicos (Liang, 2013), etc. 


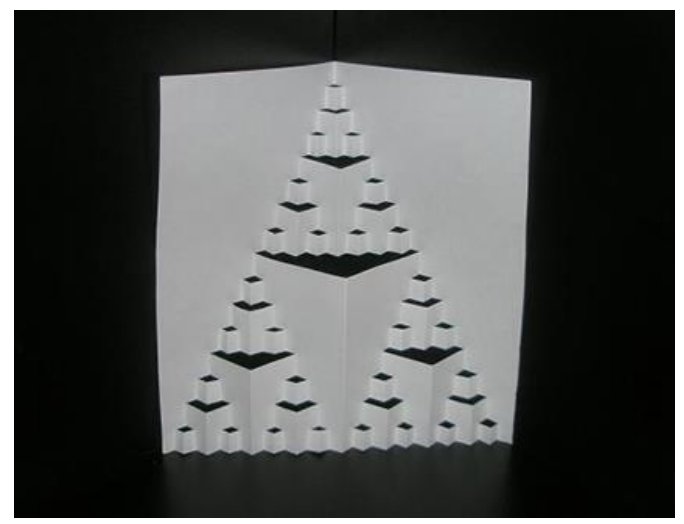

Figura 1: Matemáticas fractales en papel

Fuente: https://profmate.wordpress.com/fractales-en-papel/

En los últimos años han surgido múltiples aplicaciones y programas que crean los pop-ups de forma automática mediante herramientas informáticas (Harquail, Allen, \& Whiting, 2016) (Glassner, 2002) (Okamura \& Igarashi, 2009), sin embargo en la actividad descrita en este artículo se realizaron los diseños de las tarjetas sin utilizar procesos de composición automatizada, para que los alumnos comprendieran los principios del pop-up.

\subsection{Máquinas de corte en entornos escolares}

Tradicionalmente, para la realización de las figuras pop-up, se usan papeles de colores, plantillas, huesos de plegado y herramientas de corte como por ejemplo: tijeras, cúter, cuchillas, cizallas de corte, bases de corte magnético, cuchillos especiales, guillotinas, etc. Actualmente existen máquinas de corte digitales que pueden sustituir esas herramientas. Dentro de la fabricación digital existen dos formas constructivas: mediante la adición de material, conocida como fabricación aditiva y la segunda mediante la supresión del material o fabricación sustractiva. Cada una de las dos tiene diferentes formas y variantes, según el trabajo que se realice. En entornos educativos, la forma más conocida de la fabricación digital, son las impresoras 3D. Sin embargo, existen técnicas de fabricación digital sustractivas que emplean máquinas de corte. Dentro de los dispositivos de corte se encuentran las máquinas de corte láser, las fresadoras por control numérico (CNC) o los plotters de corte. Estas tecnologías se han abaratado en los últimos años, apareciendo opciones económicas orientadas a usuarios no profesionales que permiten contemplar su integración en contextos educativos (Saorín, Bonnet de León, Meier, \& de la Torre-Cantero, 2018).

Respecto a la primera de estas tecnologías, aunque, existen máquinas de corte láser "de escritorio" orientadas a la fabricación personal, su utilización en entornos educativos conlleva ciertos peligros derivados del cabezal láser y de la emanación de gases según el material que se corte, que exigen el uso de un filtro de aire. Sin embargo, algunas de estas máquinas se utilizan en centros de enseñanza secundaria en aulas específicas tipo makerspaces o fab labs. Estos laboratorios, con personas formadas en el uso de las tecnologías de fabricación digital tienen la posibilidad de integrar máquinas de corte láser a precios que van de los 600 a 5000 
euros, algo impensable hace apenas dos años. En este rango de precios se encuentran las siguientes herramientas "FABOOL Laser Mini" (fig. 2), "Mr Beam", "Full Spectrum Laser Hobby Series" o "Glowforce".

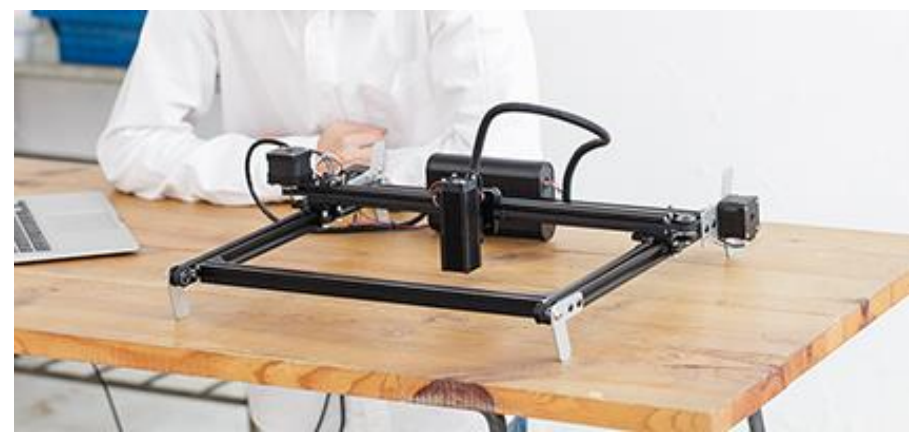

Figura 2: Máquina de corte láser: FABOOL Laser Mini

Fuente: https://www.smartdiys.cc/products/fabool-laser-mini

La segunda de las tecnologías de corte, las fresadoras, son máquinas que trabajan de forma mecanizada, tallando el material mediante el movimiento y rotación de una fresa. Tiene capacidad para cortar, desbastar, limar y afilar materiales de grandes grosores y durezas como maderas, metales, etc., en función de las especificaciones de cada marca. La tecnología de fresado y corte por control numérico también ha experimentado un fenómeno similar al de las herramientas de corte láser con la aparición de opciones de coste asequible, orientadas a la fabricación personal. Sin embargo, estas máquinas presentan algunos inconvenientes a la hora de introducirlas en entornos educativos, como el elevado nivel de ruido y polvo que producen además de la necesidad de una formación específica para el manejo de ficheros digitales y selección de parámetros de corte. A pesar de esto, están surgiendo modelos didácticos y orientados al hobby que están reduciendo cada vez más este tipo de inconvenientes. Entre estos modelos podemos encontrar propuestas como "Carbide 3D", "Carvey", "MillRight CNC", "X-Carve" o "Stepcraft" (fig. 3).

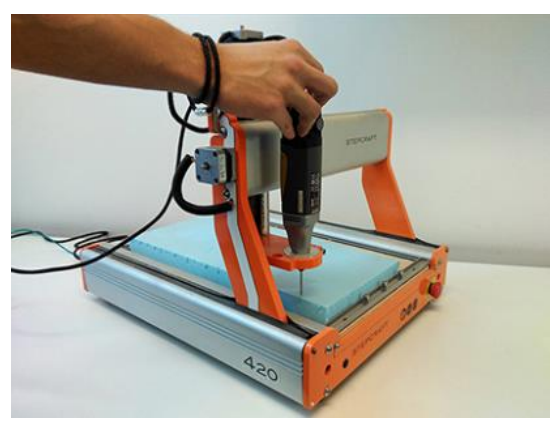

Figura 3: Fresadora: Stepcraft 420

Otra tecnología de fabricación digital es el plotter de corte. Este tipo de máquinas, dispone de un cabezal con una cuchilla que se desplaza por la superficie del material que se desea cortar o troquelar. A nivel profesional se suele utilizar en la industria gráfica y el material con el que 
se trabaja más habitualmente es el vinilo, aunque existe la posibilidad de hacerlo con gran variedad de materiales. Por un precio comprendido entre 1000 a 2000 euros, se puede adquirir un plotter de corte con características profesionales. Sin embargo, por unos 250 euros, existen plotters de corte que se pueden adquirir en papelerías orientadas al fenómeno del scrapbooking (personalización de álbumes mediante recortes). Estas máquinas no mucho mayores que una impresora de papel, son de sencillo funcionamiento y permiten cortar una amplia variedad de materiales como cartulina, cartón, tela, goma eva, etc. y son perfectamente compatibles con la mayoría de ordenadores y archivos de imágenes con los que se suele trabajar en entornos escolares. Dentro de estos dispositivos podemos encontrar modelos como Cameo y Curio (fig. 4) de la empresa Silhouette, la Cricut, la serie Stika de la empresa Roland, o ScanNcut de Brother entre otras. Algunas de ellas se venden con una bolsa de transporte con ruedas que permite desplazarlas con mucha comodidad.

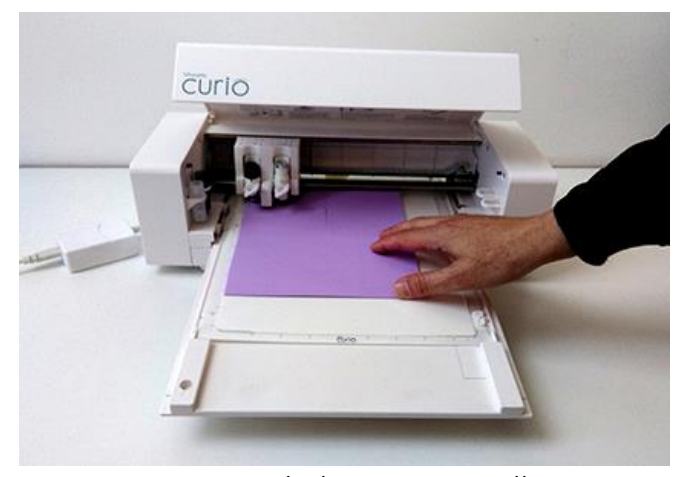

Figura 4: Curio de la empresa Silhouette

En el ámbito educativo, se valoran las herramientas de funcionamiento simple, que no requieran de una instalación compleja, que se pueda transportar con facilidad, que no produzcan residuos y que sea asequible. En este sentido, tal y como hemos visto, la mejor alternativa entre las máquinas de corte, es el plotter de corte de bajo coste. Esta herramienta es útil para cortar elementos de pequeño y medio grosor, desde papel, cartulina, goma eva, acetato, etc. Corta a una velocidad mayor que a mano, genera poco ruido, no suelta restos de material en forma de residuos u humos tóxicos y no es peligroso de usar. Teniendo en cuenta estas características, se postula como una herramienta adecuada para su utilización en entornos escolares. Los cortes que se producen con el plotter, se relacionan con los mismos procesos que se pueden realizar con cutter o tijera, pero el resultado digital suele ser más preciso y rápido.

Antes de la experiencia descrita en este trabajo, el plotter de corte se ha utilizado en el curso 2015-2016 en otro trabajo de investigación para la realización de retratos tridimensionales en entornos educativos. En esta experiencia los participantes confirmaban el interés de este tipo de actividades para la docencia de contenidos de asignaturas de expresión plástica y visual e indicaban que habían aprendido nuevas formas de construcción de figuras tridimensionales (Saorín, Bonnet de León, Meier, \& de la Torre-Cantero, 2018). El resultado de este trabajo ha 
servido para continuar la investigación sobre nuevas formas de utilizar esta herramienta de fabricación digital en entornos educativos.

\subsection{Diseño digital de las tarjetas Pop-up}

Para poder cortar con un plotter de corte, cortadora láser o fresadora es necesario disponer de un archivo digital que puede ser interpretado por la máquina. Estos archivos son generalmente conocidos como dibujos vectoriales. Éstos están formados por objetos geométricos como puntos, segmentos, polígonos, arcos o líneas. Cada uno de ellos tiene propiedades matemáticas de forma, posición, etc. que pueden ser leídas por máquinas de control numérico (Kalpakjian \& Schmid, 2002). Los formatos habituales de gráficos vectoriales son el svg (scalable vector graphic) o dxf (Drawing Exchange Format), por lo que al exportarlo se utilizará esas extensiones.

Algunos programas o aplicaciones no permiten la exportación en estos formatos por lo que es necesario utilizar un programa para cambiarlo. Actualmente existen diversos recursos online gratuitos para realizar esta acción, como por ejemplo convertio, cloudconvert, aconvert, etc.

La vectorización se trabaja en muchas disciplinas artísticas como el diseño o la ilustración. Existen una serie de programas, aplicaciones, etc. que permiten el dibujo vectorial. Se dividen en programas para ordenador, en aplicaciones para dispositivos como tabletas o móviles y programas online que normalmente funcionan en diversos dispositivos. Algunos programas permiten realizar la vectorización de forma manual y otros la realizan de forma automática a partir de una imagen o fotografía. Los programas que son para descargar e instalar en ordenadores como por ejemplo Illustrator o Autocad suelen ser los más potentes para trabajar, sin embargo son complicados de aprender, requieren una instalación y pueden entrañar un desembolso importante. Las aplicaciones para tabletas o móviles como Skedio, Vector Art Studio o Imaengine Vector deben descargarse en los dispositivos y permiten trabajar la vectorización de forma eficiente, siendo una alternativa interesante a los programas de ordenador.

Existe otra opción de programas de vectorización que minimizan algunos de los problemas vistos anteriormente. Dichos programas no requieren instalación ya que funcionan online, y aunque no tienen todas las funciones de programas profesionales como Illustrator, permiten realizar un vectorizado básico de manera muy sencilla e intuitiva. Entre estos programas online, podemos destacar Gravit designer, Vecteezy Editor o Vectr. Estos programas, al ser online, requieren conexión a internet, pero son una opción viable y gratuita, muy interesante, sobre todo en entornos educativos, donde normalmente es complicado instalar un programa sin el permiso de los administradores. En este trabajo se ha utilizado Gravit designer como programa de vectorización.

\section{MATERIALES Y MÉTODOS}




\subsection{Participantes}

La actividad se ha llevado a cabo en el Colegio San Isidro, Los Salesianos de la Orotava, Tenerife en 4 으 de la ESO en la asignatura de expresión plástica y visual. Han participado un total de 63 alumnos. Dichos alumnos rellenaron un cuestionario de conocimientos previos para valorar sus nociones sobre las tecnologías a emplear y su conocimiento sobre las tarjetas pop-up. De dicho cuestionario se extrajeron los siguientes datos:

- El 76,19\% de los participantes no tiene conocimientos de diseño vectorial.

- El 92,96\% no ha utilizado nunca un plotter de corte.

- El $77,77 \%$ no sabe lo que es una tarjeta Popup.

\subsection{Hardware y software}

Se facilitaron cartulinas blancas, lápices de colores y rotuladores para todo el grupo. El software y el hardware utilizado para la prueba piloto fue el siguiente: Como programa de vectorización, la aplicación gratuita online Gravit designer (https://designer.gravit.io/) (fig. 5). Dicha aplicación se utilizó en una sala de ordenadores del instituto con conexión a internet.

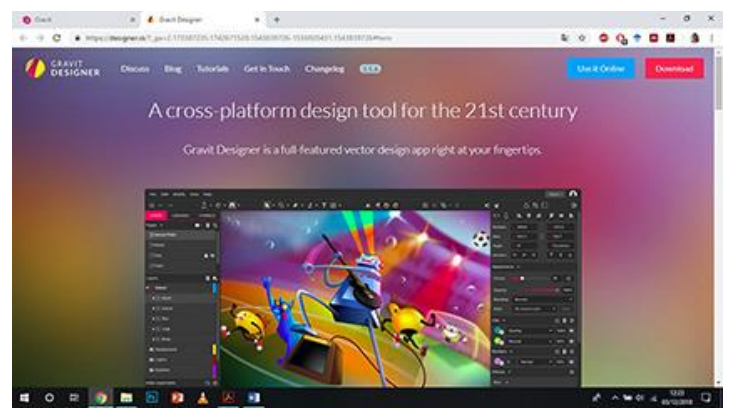

Figura 5: aplicación online Gravit

Fuente: https://designer.gravit.io/

Como herramienta digital para automatizar el corte de las cartulinas, se utilizó un plotter de corte de bajo coste de la maca Silhouette Cameo (300 €) con sus accesorios (fig. 6) y el programa gratuito Silhouette Studio 4.1 Basic Edition.

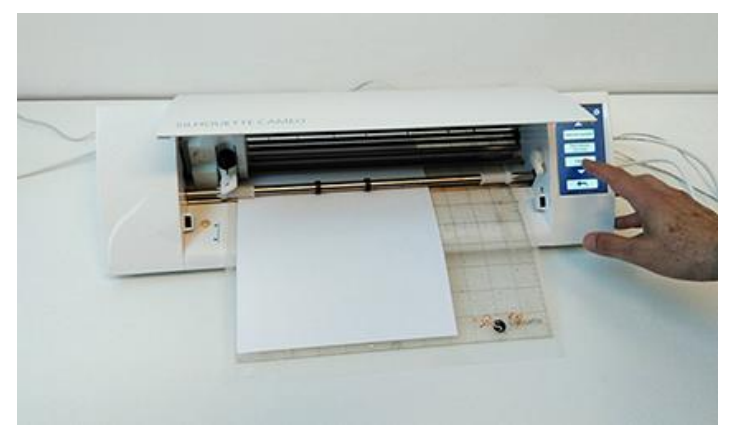

Figura 6: Plotter de corte de bajo coste de la maca Silhouette Studio 


\subsection{Experiencia realizada}

La experiencia realizada consistió en generar tarjetas pop-up de figuras de las películas de Pixar de Toy Story. Esta actividad se enmarca dentro de la asignatura de Educación Plástica, Visual y Audiovisual ya que trabaja los contenidos propios de esta asignatura de acuerdo al currículo de 4 ㅇ de la ESO. Entre los contenidos de esta asignatura destacan la elaboración imágenes digitales utilizando distintos programas de dibujo por ordenador, el uso de las TIC como medio de ayuda a la creación de proyectos y la utilización de diferentes soportes, materiales y técnicas analógicas y digitales. (Gobierno de Canarias, 2018).

Para la realización de la misma, los participantes, primero buscaban imágenes en internet de Toy Story, vectorizaban los perfiles de las figuras para poder generar una tarjeta pop-up y cortaban la misma mediante el plotter de corte (fig. 7). Por último también se animó a los alumnos a pintar las tarjetas con lápices o rotuladores de colores. La actividad se desarrolló en el colegio durante 4 sesiones lectivas de 55 minutos cada una.

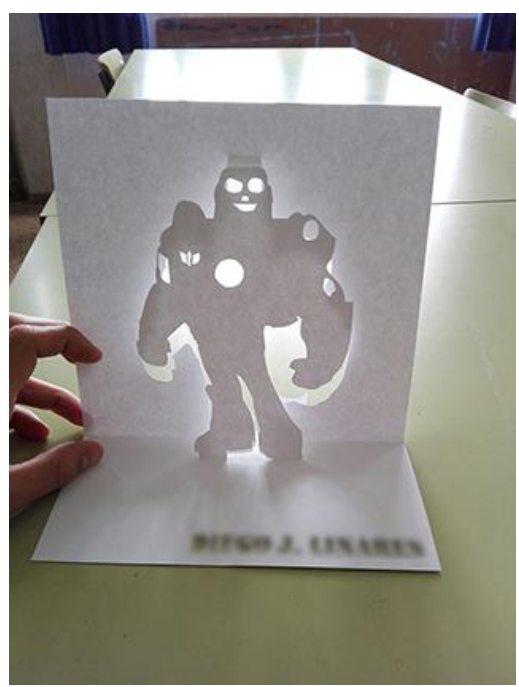

Figura 7: Ejemplo del ejercicio terminado

Para realizar dicha actividad los alumnos eligen una imagen en internet que esté relacionada con el mundo de "Toy Story" y que quieren convertir en una tarjeta pop-up. Trabajando en una sala de ordenadores se conectan al programa online Gravit para vectorizar la imagen elegida. La vectorización consiste en redibujar con la herramienta Path o pluma el contorno de la imagen elegida incluyendo también detalles como ojos, boca, etc. para que el dibujo sea reconocible. Una vez realizado todo el contorno y los detalles se exporta el archivo en formato svg.

A continuación debemos convertir el archivo .svg, en un formato dxf para poder trabajar con él en el plotter de corte. Para este paso se utilizó el programa online y gratuito convertio vector https://convertio.co/es/vector-converter/. 
Una vez que disponemos del dibujo vectorizado en formato dxf, pasamos ese archivo al ordenador que está conectado al plotter de corte Silhouette. Se importa en el programa Silhouette Studio o se arrastra el archivo a la interfaz del programa. La interfaz del programa muestra el tapete situado sobre una cuadrícula, lo que simula el espacio de corte con dicho tapete. Cuando esté la imagen del vector situada en la interfaz, se modifica el tamaño, se realizan los arreglos necesarios, añadiendo las solapas para convertir el dibujo en un pop-up y se convierten las líneas en líneas punteadas de aquellas partes del vector que no queremos que recorte el plotter porque es donde se realizarán los pliegues. Dichos pliegues permiten luego sacar la tercera dimensión al doblar la tarjeta. Por otro lado, en la actividad, cada alumno añadió su nombre a su tarjeta. Para ello existe la herramienta de texto en el programa, cuyo símbolo es una A mayúscula.

Antes de cortar es necesario preparar los ajustes de corte de la máquina Silhouette Studio 4.1 Basic Edition. En el mismo programa modifican los parámetros necesarios de corte según el material, en este caso, será cartulina. Este material aparece en los parámetros predeterminados de corte y nos indica a qué altura debe estar la cuchilla.

A continuación solo hay que cargar el material en el plotter y enviar el archivo para su corte (fig 8). Para terminar el alumno realiza los dobleces correctos en la cartulina cortada para que sobresalga la forma tridimensional del pop-up.

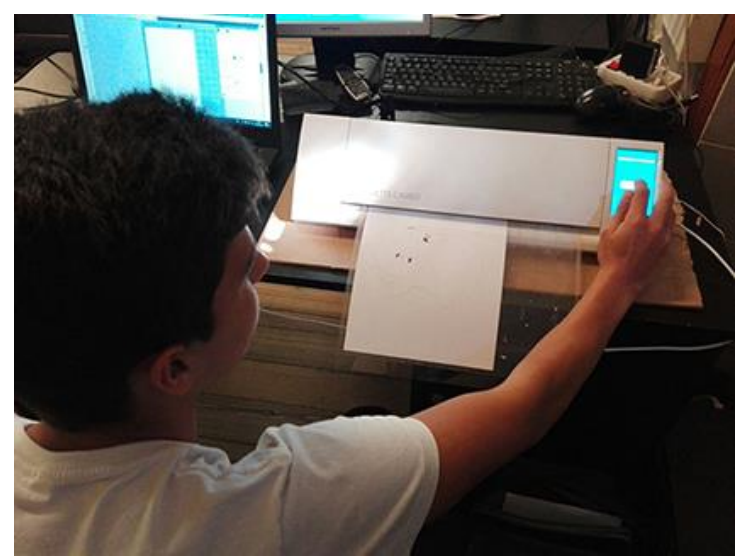

Figura 8: Alumno preparando el plotter de corte para realizar su tarjeta

Por último se realizan los dobleces en las tarjetas pop-up para conseguir la tercera dimensión (fig. 9). 


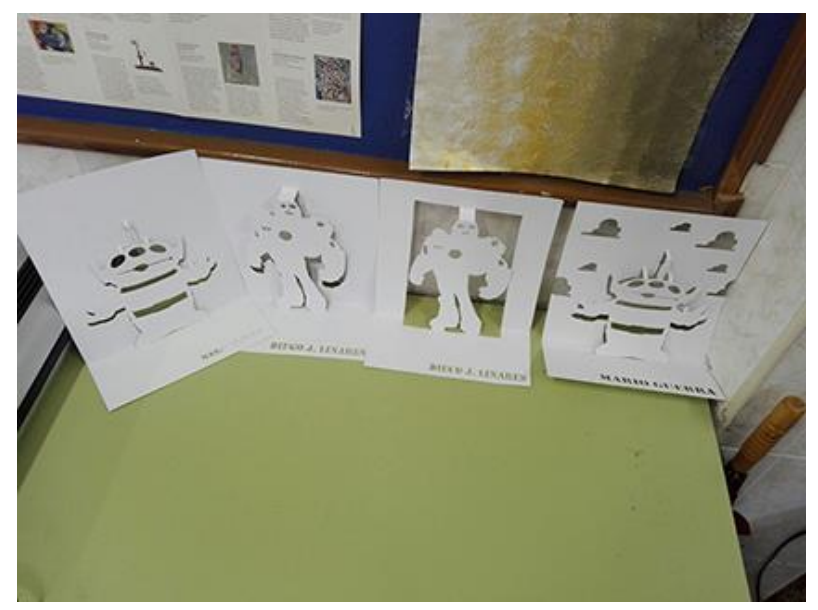

Figura 9: Resultados obtenidos por los alumnos

\subsection{Instrumentos de medida}

Como instrumento de medida se han utilizado dos cuestionarios de satisfacción. Dichos cuestionarios han sido revisados y validados por cuatro expertos en el campo de las tecnologías de fabricación digital en educación. Se ha utilizado un cuestionario previo a la actividad para valorar la idea inicial que tenían los alumnos sobre la actividad y otro cuestionario posterior para valorar la aceptación de la actividad por parte del alumno. Ambos cuestionarios se valoran según la escala Likert, donde hay cinco opciones de respuesta: nada de acuerdo, poco de acuerdo, de acuerdo, bastante de acuerdo y totalmente de acuerdo. En cada uno de los cuestionarios, y para ver el efecto que tiene la actividad en el aprendizaje de los elementos básicos de esta actividad, se les pregunta si conocen las tecnologías de vectorización, el plotter de corte y las tarjetas pop-up. Dichas preguntas se responden con sí o no.

Las preguntas del Cuestionario previo a la actividad son las siguientes:

- Creo que la vectorización y corte a través de máquinas tiene sentido dentro de mi formación estudiantil.

- Creo que crear tarjetas pop-up en 3D es difícil.

- Prefiero el aprendizaje con medios tradicionales frente a los digitales.

- Los materiales digitales son un buen complemento para mi formación.

- Creo que tengo los conocimientos necesarios para vectorizar.

- El uso de tecnologías de fabricación digital aumenta mi motivación.

Las preguntas del cuestionario posterior a la actividad son las siguientes:

- Me resultó fácil vectorizar imágenes:

- Me siento satisfecho con el resultado obtenido (tarjeta pop-up):

- He aprendido a utilizar nuevas herramientas digitales:

- Prefiero realizar pop-ups utilizando herramientas digitales frente a tradicionales.

- He obtenido la información necesaria para realizar pop-up por cuenta propia. 
- Recomendaría la actividad a otras personas.

- Creo que el plotter de corte es muy interesante para realizar trabajos propios.

- Creo que otras asignaturas podrían utilizar las máquinas de corte.

\section{RESULTADOS}

\section{Cuestionario inicial}

De dicho cuestionario se extrajeron los siguientes datos sobre los elementos básicos de esta actividad:

- El $76,19 \%$ de los participantes no tiene conocimientos de diseño vectorial.

- El 92,96\% no ha utilizado nunca un plotter de corte.

- El $77,77 \%$ no sabe lo que es una tarjeta Popup.

En la tabla 1 se muestran los resultados del cuestionario de satisfacción previo a la actividad:

Tabla 1: Resultados del cuestionario de satisfacción previo

\begin{tabular}{llc}
\hline & \multicolumn{1}{c}{ Pregunta } & $\begin{array}{c}\text { Media } \\
\text { (Des est.) }\end{array}$ \\
\hline $\begin{array}{l}\text { Creo que la vectorización y corte a través de máquinas tiene sentido dentro de mi } \\
\text { formación. }\end{array}$ & 2,33 \\
\hline 2 & Creo que crear tarjetas pop-up en 3D es difícil. & $3,28)$ \\
\hline \multirow{2}{*}{3} & Prefiero el aprendizaje con medios tradicionales frente a los digitales & $(0,89)$ \\
\hline 4 & Los materiales digitales son un buen complemento para mi formación. & 2,33 \\
\hline \multirow{2}{*}{5} & Creo que tengo los conocimientos necesarios para vectorizar. & $(1,06)$ \\
\hline \multirow{2}{*}{6} & \multirow{2}{*}{ El uso de tecnologías de fabricación digital aumenta mi motivación. } & 3,29 \\
& & $(1,11)$ \\
\hline
\end{tabular}

\section{Cuestionario posterior}

De dicho cuestionario se extrajeron los siguientes datos sobre los elementos básicos posteriores a la realización de esta actividad:

- El $96.80 \%$ de los participantes ha aprendido a realizar diseño vectorial.

- El $87,50 \%$ ha aprendido a manejar el plotter de corte.

- El $87,50 \%$ ha aprendido el concepto y a crear una tarjeta Popup.

En la tabla 2 se muestran los resultados del cuestionario de satisfacción después de realizar la actividad: 
Tabla 2: Resultados del cuestionario de satisfacción posterior

\begin{tabular}{|c|c|c|}
\hline & Pregunta & $\begin{array}{c}\text { Media } \\
\text { (Des est.) }\end{array}$ \\
\hline 1 & Me resultó fácil vectorizar imágenes & $\begin{array}{c}3,84 \\
(1,05) \\
\end{array}$ \\
\hline 2 & Me siento satisfecho con el resultado obtenido (tarjeta pop-up). & $\begin{array}{c}3,84 \\
(1,02) \\
\end{array}$ \\
\hline 3 & He aprendido a utilizar nuevas herramientas digitales & $\begin{array}{c}4,00 \\
(0,84)\end{array}$ \\
\hline 4 & Prefiero realizar pop-ups utilizando herramientas digitales frente a tradicionales & $\begin{array}{c}3,97 \\
(0,86) \\
\end{array}$ \\
\hline 5 & He obtenido la información necesaria para realizar pop-up por cuenta propia & $\begin{array}{c}4,13 \\
(0,71)\end{array}$ \\
\hline 6 & Recomendaría la actividad a otras personas & $\begin{array}{c}4,13 \\
(0,71)\end{array}$ \\
\hline 7 & Creo que el plotter de corte es muy interesante para realizar trabajos propios & $\begin{array}{c}3,75 \\
(0,88)\end{array}$ \\
\hline 8 & Creo que otras asignaturas podrían utilizar las máquinas de corte & $\begin{array}{c}3,59 \\
(0,98)\end{array}$ \\
\hline
\end{tabular}

\section{CONCLUSIONES}

Los resultados en la experiencia demuestran que la fabricación digital mediante plotter de corte y el diseño vectorial mediante programas digitales online resultan viables en educación secundaria sin necesidad de crear un Fab Lab (laboratorio de fabricación digital) en el centro educativo. El plotter utilizado que dispone de un maletín con ruedas resulta muy cómodo para desplazarse. Por otro lado, respecto a la dificultad y fiabilidad de esta herramienta, podemos afirmar que el plotter de corte de la marca Silhouette Cameo 2 ha funcionado correctamente sin generar problemas ni a los alumnos ni al profesor, ya que en torno a las 100 cortes realizadas (entre pruebas previas, ejercicio y errores), no produjo ningún fallo propio de la máquina. Los problemas que se detectaron estaban relacionados con los materiales adicionales, como el tapete y el desgaste de la cuchilla. Por lo tanto, tanto su precio como su funcionamiento es óptimo para el trabajo en centros educativos.

La actividad realizada en el marco de la asignatura de Educación Plástica, Visual y Audiovisual trabaja los contenidos propios de esta asignatura como por ejemplo el uso de las TIC como medio de ayuda a la creación de proyectos. La utilización de diferentes soportes, materiales y técnicas analógicas y digitales. Y la elaboración imágenes digitales utilizando distintos programas de dibujo por ordenador. Por lo tanto es una actividad que encaja a la perfección en la asignatura y trabaja varios contenidos.

Aunque la actividad se puede desarrollar también con herramientas manuales como tijeras y cutter, destacamos el uso de las nuevas tecnologías en el aula para fomentar la competencia digital entre los alumnos. 
De acuerdo a los resultados del cuestionario previo y posterior, podemos concluir que introducir la vectorización y corte permite trabajar las competencias digitales en la asignatura de expresión plástica y visual y genera satisfacción entre los participantes. Los estudiantes manifiestan no solo que han aprendido nuevas herramientas digitales (4/5) sino que se recomendarían las tecnologías de corte para otras asignaturas (3,59/5). Además, los alumnos manifiestan que se sientan satisfechos con el resultado obtenido $(3,84 / 5)$.

Al comparar los resultados del cuestionario inicial con el posterior a la actividad podemos concluir que los participantes han aprendido a realizar dibujos vectoriales y manifiestan que además han aprendido a manejar el plotter de corte. Para ello han aprendido el concepto de tarjeta pop-up y así como su proceso de creación.

Respecto al uso de tecnologías digitales, se observa que entre los estudiantes aumentó la preferencia de aprender con métodos digitales frente a los tradicionales (2,33 en el cuestionario inicial frente a 3,97 en el posterior). Además los alumnos opinan que han recibido los conocimientos necesarios para realizar un trabajo de vectorización de forma autónoma (4,13 sobre 5$)$ y además recomendarían la actividad a otras personas (4,13 sobre 5$)$.

El trabajo de las tarjetas pop-up ofrece múltiples opciones y temáticas. En este trabajo se ha restringido la forma y el pliegue frontal para cada uno, variando solo el contenido o la imagen de la tarjeta. Para futuras actividades, los pliegues pueden variar al igual que el tipo de corte. También se propone ampliar las líneas temáticas para usar la creación de pop-ups mediante plotter de corte en ámbitos de patrimonio, realizando el trabajo sobre diferentes edificios relevantes, realizar trabajos sobre esculturas, etc. Por otro lado se plantea estudiar, si este tipo de ejercicios estimula la creatividad y la motivación de los participantes.

\section{REFERENCIAS}

Alvarado, R. G. (2012). Fabricación digital de modelos constructivos: análisis de equipos y procesos. Revista Facultad de Ingeniería(59), 145-157.

Blees, M., Barnard, A., Rose, P., Roberts, S., McGill, K., Huang, P., . . McEuen, P. (2015). Graphene kirigami. Nature(524(7564)), 204-207. doi:https://doi.org/10.1038/nature14588

BOC. (13 de Julio de 2016). DISPOSICIONES GENERALES - Consejería de Economía, Industria, Comercio y Conocimiento. Boletín Oficial de Canarias núm. 134. Canarias, España. Obtenido de http://www.gobiernodecanarias.org/boc/2016/134/003.html

Bonnet de León, A., Meier, C., Saorín, J., de la Torre-Cantero, J., \& Carbonell, C. (2017). Tecnologías de diseño y fabricación digital de bajo coste para el fomento de la competencia creativa. Arte, Individuo y Sociedad, 29(1), 85-100. doi:http://dx.doi.org/10.5209/ARIS.51886 
Bromberg, H. M. (1995). Washington Patente no 5,450,680.

Carter, D., \& Díaz, J. (1999). The elements of pop-up: A pop-up bookfor aspiring paper engineers. United States: Little Simon.

Castillo Cordova, L. (2016). Kirigami y habilidades creativas en estudiantes de las facultades de educación. tesis. Huancayo, Perú: Universidad nacional del centro de Perú.

de la Torre-Cantero, J., Saorín, J., Meier, C., Melián Díaz, D., \& Alemán, M. (2015). Creación de réplicas de patrimonio escultórico mediante reconstrucción 3D e impresoras 3D de bajo coste para uso en entornos educativos. Arte, Individuo y Sociedad, 27(3), 427-444.

Europa Press. (30 de 11 de 2018). Los centros gallegos contarán el curso que viene con un Bachillerato de excelencia en ciencias y tecnologías. Obtenido de https://www.europapress.es/galicia/noticia-centros-gallegos-contaran-curso-vienebachillerato-excelencia-ciencias-tecnologias-20180611164944.html

Freeman, A., Becker, S., \& Cummins, M. (2017). NMC/CoSN Horizon Report: K-12 Edition. United States: The New Media Consortium.

García Cuéllar, D. J., Flores Salazar, J. V., \& Martínez Miraval, M. (2017). Génesis instrumental de la noción de fractal en docentes de matemáticas de nivel secundario. VIII CONGRESO IBEROAMERICANO DE EDUCACIÓN MATEMÁTICA. LIBRO DE ACTAS (págs. 455-463). Madrid: FESPM.

Glassner , A. (2002). Interactive pop-up card design. IEEE Computer Graphics and Applications (págs. 22(1), 79-86). IEEE. doi:DOI: 10.1109/38.974521

Gobierno de Canarias. (30 de 11 de 2018). Consejería de Educación y Universidades. Obtenido de Programa STEAM: Fomento de Vocaciones Científicas y Creatividad: http://www.gobiernodecanarias.org/educacion/web/programas-redeseducativas/programas-educativos/steam/

Gobierno de Canarias. (2018). Currículos de las materias de la Educación Secundaria Obligatoria. Canarias: Consejería de educación y Universidades. Obtenido de http://www.gobiernodecanarias.org/educacion/web/secundaria/informacion/ordenac ion-curriculo/curriculos_eso_lomce.html

Harquail, N., Allen, M., \& Whiting, E. (2016). Foldlings: a tool for interactive pop-up card design. Eurographics Workshop on Graphics for Digital Fabrication (págs. 31-38). Lisbon: Eurographics Association.

Isobe, M., \& Okumura, K. (2016). Initial rigid response and softening transition of highly stretchable kirigami sheet materials. Scientific reports(6, 24758). doi:https://doi.org/10.1038/srep24758 
Jablonski, R. (1976). The paper cut-out design book: a sourcebook for creating and adapting the heritage of American folk art, Polish wycinanki, Chinese hua yang, Japanese kirigami, German Scherenschnitte, and others. Owings Mills: Stemmer House.

Kalpakjian, S., \& Schmid, S. (2002). Manufactura, ingeniería y tecnología. México: Pearson Educación.

Liang, Y. (2013). Applying kirigami models in teaching micro-electro-mechanical systems. 3rd Interdisciplinary Engineering Design Education Conference (págs. 83-86). Santa Clara, California: IEEE. doi:https://doi.org/10.1109/IEDEC.2013.6526765

McCormick, C., \& White, K. (2011). Folklore: EL (Vol. 2). California: ABC-CLIO.

Monteiro Kobayashi, M., \& Ueno Yamada, T. (2013). Origami e kirigami: arte e cultura como recurso lúdico e educativo. Revista Ciência em Extensão, 9(3), 148-158.

Nogueira Valente, V., \& Yukari Ota, C. (2015). The art of origami, kirigami and origamic architecture helping improve the spatial abilities. VIII World Congress on Communication and Arts (págs. 279-283). Salvador, Brasil: COPEC.

Okamura, S., \& Igarashi, T. (2009). An interface for assisting the design and production of popup card. International Symposium on Smart Graphics (págs. 68-78). Berlin, Heidelberg: Springer, Berlin. doi:https://doi.org/10.1007/978-3-642-02115-2_6

Romero Velasco, O., \& Tovar Cifuentes, A. (2018). Herramienta Didáctica Basada en Kirigami para Contribuir en el Desarrollo del Pensamiento Creativo, Implementada en Estudiantes de Grado Décimo de una Institución Educativa Distrital. Tesis. Bogotá: Universidad distrital Francisco José de Caldas.

Saorín, J., Bonnet de León, A., Meier, C., \& de la Torre-Cantero, J. (2018). Retrato tridimensional mediante la utilización de tecnologías de fabricación digital de bajo coste en entornos educativos. Arte, Individuo y Sociedad, 30(2), 295-309. doi:http://dx.doi.org/10.5209/ARIS.56796

Saorín, J., de la Torre-Cantero, J., Bonnet de León, A., \& Meier, C. (2016). Creación de objetos tridimensionales de cartón mediante tecnologías de bajo coste como alternativa a la impresión 3D en entornos educativos. Comunicación y pedagogía: Nuevas tecnologías y recursos didácticos, (291), 18-23.

Sareh, S., \& Rossiter, J. (2012). Kirigami artificial muscles with complex biologically inspired morphologies. Smart Materials and Structures, 22(1)(014004). doi:https://doi.org/10.1088/0964-1726/22/1/014004

Sen, K. C. (1721). Wakoku Chiyekurabe (Mathematical Contests). Japan: http://theory. Ics. mit. edu/ edemaine/foldcut/sen_book. html. 
Shyu, T., Damasceno, P., Dodd, P., Lamoureux, A., Xu, L., Shlian, M., . . Kotov, N. (2015). A kirigami approach to engineering elasticity in nanocomposites through patterned defects. Nature Materials, 14, 785-789. doi:https://doi.org/10.1038/nmat4327

Temko, F. (1962). Kirigami: The Creative Art of Paper Cutting. New York: Platt \& Munk.

Vyzoviti, S. (2006). Supersurfaces: Folding as a method of generating forms for architecture, products and fashion. Bis Pub.

\section{Para referenciar este artículo:}

Bonnet de León, A., Saorin, J., de la Torre-Cantero, J., Meier, C., \& García Marrero, E. (2019). Diseño y fabricación digital de tarjetas pop-ups en entornos educativos mediante tecnologías de bajo coste. Edutec. Revista Electrónica De Tecnología Educativa, (67), 48-65. https://doi.org/10.21556/edutec.2019.67.1261 